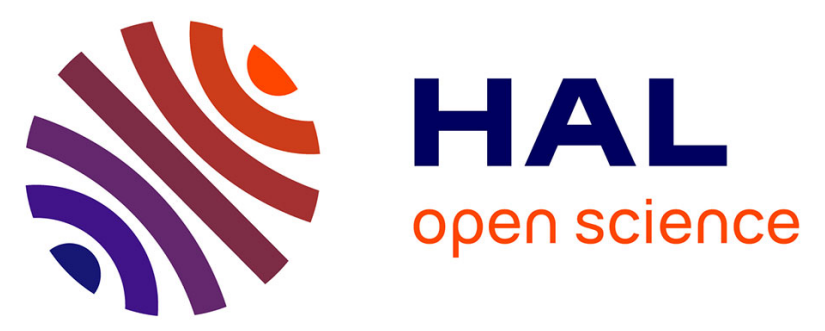

\title{
Density effect of gold nanodisks on the SERS intensity for a highly sensitive detection of chemical molecules
}

Jean-François Bryche, Raymond Gillibert, Grégory Barbillon, Mitradeep

Sarkar, Anne-Lise Coutrot, Frédéric Hamouda, Abdelhanin Aassime, Julien

Moreau, Marc Lamy de La Chapelle, Bernard Bartenlian, et al.

\section{To cite this version:}

Jean-François Bryche, Raymond Gillibert, Grégory Barbillon, Mitradeep Sarkar, Anne-Lise Coutrot, et al.. Density effect of gold nanodisks on the SERS intensity for a highly sensitive detection of chemical molecules. Journal of Materials Science, 2015, 50 (20), pp.6601-6607. 10.1007/s10853-015-9203-x . hal-01230274

\section{HAL Id: hal-01230274 \\ https://hal-iogs.archives-ouvertes.fr/hal-01230274}

Submitted on 5 Oct 2017

HAL is a multi-disciplinary open access archive for the deposit and dissemination of scientific research documents, whether they are published or not. The documents may come from teaching and research institutions in France or abroad, or from public or private research centers.
L'archive ouverte pluridisciplinaire HAL, est destinée au dépôt et à la diffusion de documents scientifiques de niveau recherche, publiés ou non, émanant des établissements d'enseignement et de recherche français ou étrangers, des laboratoires publics ou privés. 


\title{
Density effect of gold nanodisks on the SERS intensity for a highly sensitive detection of chemical molecules
}

\author{
Jean-François Bryche • Raymond Gillibert • Grégory Barbillon • \\ Mitradeep Sarkar • Anne-Lise Coutrot • Frédéric Hamouda • \\ Abdelhanin Aassime • Julien Moreau - Marc Lamy de la Chapelle . \\ Bernard Bartenlian • Michael Canva
}

Received: date / Accepted: date

\begin{abstract}
Surface Enhanced Raman Scattering is a sensitive and widely used as spectroscopic technique for chemical and biological structure analysis. One of the keys to increase the sensitivity of SERS sensors is to use nanoparticles/nanostructures. Here, we report on the density effect of gold nanodisks on SERS intensity for a highly sensitive detection of chemical molecules. Various densities of gold nanodisks with a height of 30 $n m$ on gold/glass substrate were fabricated by electron beam lithography in order to have a good uniformity and reproducibility. The evolution of the Enhancement Factor with nanodisk density was quantified and compared to numerical calculations. An enhancement factor as high as $2.6 \times 10^{7}$ was measured for the nanodisk with a diameter of $110 \mathrm{~nm}$ and a periodicity of $150 \mathrm{~nm}$ which corresponds to the biggest density $(42.2 \%)$.
\end{abstract}

Keywords Surface Plasmons · E-beam Lithography · Biosensors · SERS · Plasmonics

\section{Introduction}

Surface Enhanced Raman spectroscopy (SERS) is a powerful technique of molecular detection. The SERS effect

Jean-François Bryche · Grégory Barbillon · Frédéric Hamouda · Abdelhanin Aassime · Bernard Bartenlian Institut d'Electronique Fondamentale CNRS UMR 8622, Université Paris-Sud, Bâtiment 220, 91405 Orsay Cedex, France.

E-mail: jean-francois.bryche@u-psud.fr

Mitradeep Sarkar · Anne-Lise Coutrot • Julien Moreau . Michael Canva

Laboratoire Charles Fabry CNRS UMR 8501, Institut d'Optique Graduate School, 91227 Palaiseau Cedex, France.

Raymond Gillibert · Marc Lamy de la Chapelle

Laboratoire Chimie, Structures, Propriétés de Biomatériaux et d'Agents Thérapeutiques, CNRS UMR 7244,

Université Paris-Nord, 93017 Bobigny Cedex, France. is strongly dependent on the electromagnetic (EM) field intensity. In order to compensate for the very low Raman scattering cross-sections of the target molecules, EM enhancement is necessary and can be obtained with plasmonic nanostructures. As SERS signals strongly depend on the size, the shape and the organization of the nanostructures, the uniformity, reproducibility and stability of SERS substrates with high enhancement factor are extremely important $[1,2]$. These are critical bottlenecks in the realization of industrial SERS applications. Several lithographic techniques, such as Nanoimprint Lithography (NIL) [3-5], NanoSphere Lithography (NSL) [6-8], Electron Beam Lithography (EBL) [913], X-ray Interference Lithography [14] are used to fabricate SERS substrates. Recently, we also demonstrated the presence of a hybrid plasmonic mode when a gold film under the nanocylinders is added [15]. This plasmonic mode allows a strong enhancement of the electromagnetic field intensity, and consequently a potential improvement of the SERS signal. Following this study, we report in this paper on the density effect of gold nanodisks on the SERS intensity. Experimental results are shown for nanodisk arrays of different periods and compared to numerical calculations. To ensure a good uniformity and reproducibility, electron beam lithography was used to fabricate the samples.

\section{Experimental details}

\subsection{Au nanodisk fabrication}

The fabrication process is summarized in figure 1. Main steps are (i) cleaning of the glass substrate, (ii) evaporation of titanium/gold layers under vacuum and PMMA spin-coating, (iii) E-Beam Lithography, (iv) develop- 
ment (v) second gold deposition by evaporation under vacuum, (vi) lift-off process.

Substrates are $900 \mu m$ thick D263 borosilicate glasses from Schott with a refractive index of 1.53 at $\lambda=546$ $\mathrm{nm}$ and a transmission around $90 \%$ in IR-Visible domains. The borosilicate glass is first carefully cleaned following a classical protocol, which includes solvents and $\mathrm{H}_{2} \mathrm{SO}_{4} / \mathrm{H}_{2} \mathrm{O}_{2}(3: 1)$ solution (Piranha solution). After drying, the sample is covered with $2 \mathrm{~nm} \mathrm{Ti} / 30$ $\mathrm{nm} \mathrm{Au}$ layers obtained by electron beam evapora-tion. Titanium is used here as adhesion layer.

To prepare the e-beam lithography step, PMMA A2 (polymethylmethacrylate) is spin-coated and baked on the substrate $\left(80 \mathrm{~nm}\right.$ thick). Several $100 \times 100 \mu \mathrm{m}^{2}$ and $200 \times 200 \mu \mathrm{m}^{2}$ gold nanodisk arrays with periodicities $\Lambda$ ranging from 80 to $300 \mathrm{~nm}$ and diameters $D$ of 50 and $110 \mathrm{~nm}$ are fabricated. E-Beam parameters have been carefully optimized to take in account the over-exposure resulting from the proximity effects. As the pattern density chosen is rather important, obtaining a good definition of the nanodisks (i.e. shape, dimensions and periodicity), leads to set the writing current between 2 and $2.2 \mathrm{nA}$ with a dose between 6 and $7.5 \mathrm{nC} / \mathrm{cm}^{2}$. All samples were realized with a NanoBeam (nB4) sys-tem with an accelerating voltage of $80 \mathrm{kV}$. Samples are developed in a (1:3) methylisobutylketone/isopropanol (MIBK/IPA) solution during about $2 \mathrm{~min}$, and finally rinsed with isopropanol. A $30 \mathrm{~nm}$ gold layer is then evaporated on the sample, and the nanodisks are fi-nally obtained by lift-off process in acetone. Eventually, to be sure that resist residues are completely avoided, the sample is totally cleaned by oxygen plasma during 5 min at $200 \mathrm{~W}$ power.

On figure 2, we show SEM pictures for two couples of parameters $(D=110 \mathrm{~nm}, \Lambda=150 \mathrm{~nm}$ and $D=50$ $\mathrm{nm}, \Lambda=80 \mathrm{~nm})$. The nanodisks obtained on the figure 2 show good definition with an average diameter of $113 \mathrm{~nm}$ and $52 \mathrm{~nm}$ compared to the defined values, respectively. In the same way, the average gap of $37 \mathrm{~nm}$, and $29 \mathrm{~nm}$ are obtained for these two types of patterns, respectively. No significant dispersion of these parameters was observed across the whole pattern area. We obtain for these couples of parameters, two fill-factors (or surface densities) defined by the ratio between the disk area (top) and the square of the periodicity: $\rho=$ $\left(\pi D^{2}\right) /\left(4 \Lambda^{2}\right)$. Thus, we have around $42.2 \%$ for the first couple (110-150 $\mathrm{nm}$ ) and $30.7 \%$ for the second (50-80 $\mathrm{nm}$ ) compared to the maximum density of $78.5 \%$ (for disks in contact).
Cleaned D263 Borosilicate Substrate

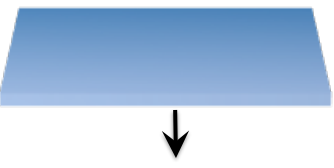

Deposition of Ti/Au evaporated layer + PMMA spin-coating
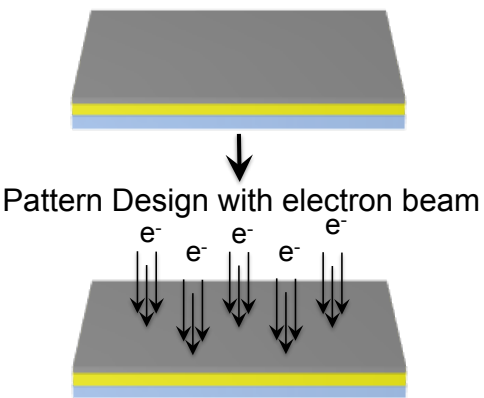

$\downarrow$

Development in solution MIBK/ISO
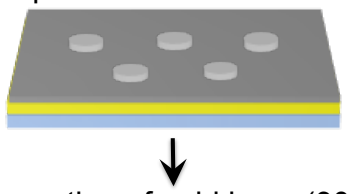

Evaporation of gold layer (30 nm)

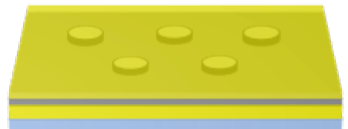

$\downarrow$

Gold disks after lift-off process in acetone

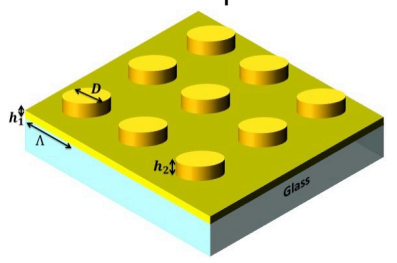

Fig. 1 Principle scheme of the fabrication of gold nanostructures used for chemical sensors, $\mathrm{h}_{1}$ and $\mathrm{h}_{2}$ represent the gold film thickness $\left(h_{1}=30 \mathrm{~nm}\right)$, and the nanostructure height $\left(h_{2}=30 \mathrm{~nm}\right)$, respectively.

\subsection{Thiophenol functionalization}

For the sample functionalization, thiophenol $\left(\mathrm{C}_{6} \mathrm{H}_{6} S\right)$ molecule is used in order to test the sensitivity of these samples as SERS substrates. The functionalization is decomposed in four steps: (i) preparation of a $0.1 \mathrm{mM}$ solution of thiophenol in ethanol, (ii) dipping the sample in the solution during $2 h$, (iii) washing thoroughly with pure ethanol, (iv) drying with nitrogen. In addition, the thiophenol concentration used for the Raman experiment is $1 M$. 

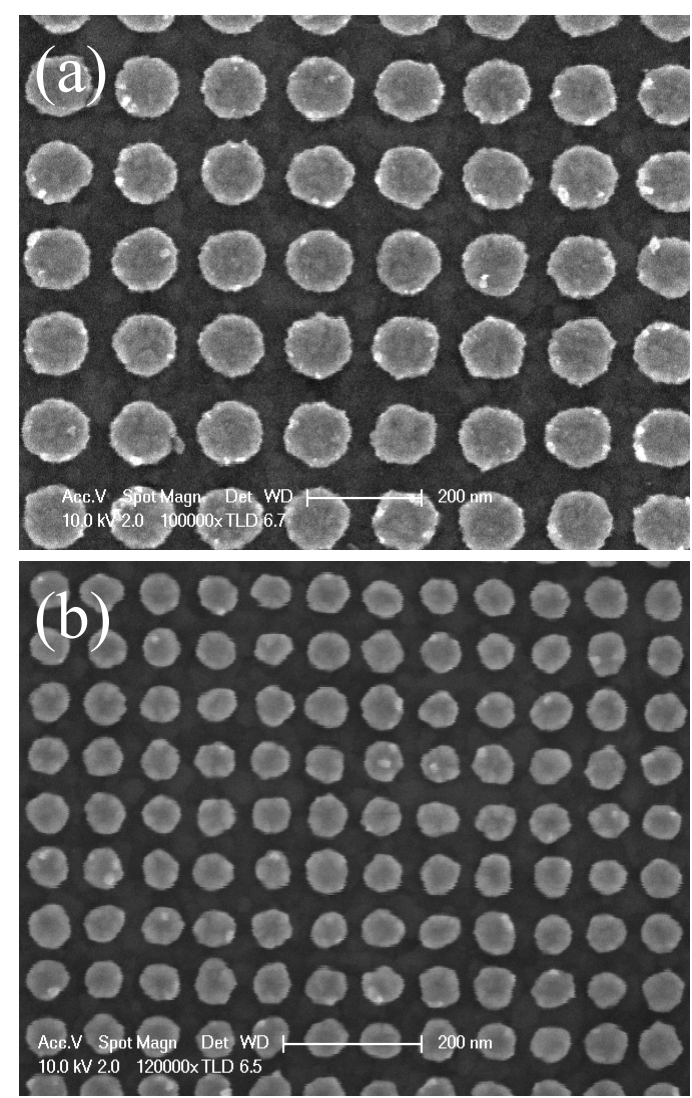

Fig. 2 SEM pictures of gold nanodisks on gold surface (scale bar $=200 \mathrm{~nm}$ ) with the following sizes : (a) $D=110 \mathrm{~nm}, \Lambda$ $=150 \mathrm{~nm}$, and (b) $D=50 \mathrm{~nm}, \Lambda=80 \mathrm{~nm}$.

\subsection{Optical characterization}

Raman spectra were recorded using a Xplora spectrophotometer from Horiba Scientific. The acquisition time was fixed to $10 \mathrm{~s}$. A $660 \mathrm{~nm}$ laser was used for all experiments with a power of $288 \mu \mathrm{W}$. The laser excitation was focused on the substrate using a microscope objective $(\times 100$, N.A. $=0.9)$. The same objective was used to collect the Raman signal from the SERS substrates in a backscattering configuration. SERS spectra were recorded with a spectral resolution under $4 \mathrm{~cm}^{-1}$. The illuminated area is around $144 \mu \mathrm{m}^{2}$. In addition, for classical Raman measurements in solution, a macroobjective with a focal length of $40 \mathrm{~mm}(\mathrm{~N} . \mathrm{A} .=0.18)$ and a $660 \mathrm{~nm}$ laser were used. All obtained spectra have been normalized by the acquisition time. We also take into account the N.A. and the laser power in order to determine the E.F.

\section{Results and discussion}

Functionalized nanodisks with the thiophenol molecules following the protocol described in the paragraph 2.2 were characterized by SERS measurements. Figure 3(1) represents the extinction spectra of nanodisks for two different couples of dimensions: (a) $D=50 \mathrm{~nm}, \Lambda=80$ $\mathrm{nm}$, and (b) $D=110 \mathrm{~nm}, \Lambda=200 \mathrm{~nm}$. The plasmonic resonances associated to these two couples of dimensions are $\lambda_{\text {Plasmon }}=520 \mathrm{~nm}, \lambda_{\text {Plasmon }}=532$ $n m$, respectively. Figures 3(2) and 3(3) represent the SERS spectra of thiophenol molecules obtained with these two samples. Characteristic Raman shifts of thiophenol molecules [16] are observed in the figures 3(2) and 3(3). No significant SERS signal is observed for the gold film (see figure 3(4e)). The highest values of SERS intensities were found for the size $D=110 \mathrm{~nm}$ and $\Lambda$ $=150 \mathrm{~nm}$ (see figure 3(3)).

On figure 3(4), we studied the effect of the grating's periodicity on the SERS intensity by keeping constant the diameter of disks $(D=110 \mathrm{~nm})$. A reduction of the SERS intensity is observed for all characteristic peaks, when the periodicity of nanodisks increases (see Table 1, for the values of the peak intensity at 1575 $\mathrm{cm}^{-1}$ ). This is explained by the reduction of the coupling between nanodisks and the consequent reduction of the electric field intensity around them. Proximity between nanodisks plays a key role for SERS signal, as well as the nanodisk size. Similar studies based on nanodisks fabricated on $\mathrm{Si}$ substrates have been reported before. Such reports have demonstrated SERS enhancement either by structures fabricated by X-ray interference lithography [14] with small gap or by simulation [18]. However in such structures with a metallic array on glass or Si substrates, only the localized plasmon resonance (LSPR) can be exctited. Thus, the reported enhancement results from the confinement of the electromagnetic field by such LSPR modes. The structure and the physical phenomenon studied in this article is however different from the above mentioned citation, in the fact that the structure studied here consists of an underlying uniform metallic layer along with the metallic array. This uniform layer can support the propagating surface plasmon (PSP) resonance. The PSP owing to the periodicity of the structure can couple to give rise to hybrid modes $[15,17]$. Such hybrid modes of-fer a greater enhancement of the electromagnetic field around the metallic cylinders. Thus, the SERS inten-sity obtained by using an array of metallic cylinders with an underlying uniform metallic film is higher than using the array on $\mathrm{Si}[14,19]$ or only on glass [20] sub-strates.

Moreover, to compare efficiency of our substrates, we evaluate the Enhancement Factor (EF), which is defined as follows:

$E F=\frac{I_{S E R S}}{I_{\text {Raman }}} \times \frac{N_{\text {Raman }}}{N_{\text {SERS }}}$ 

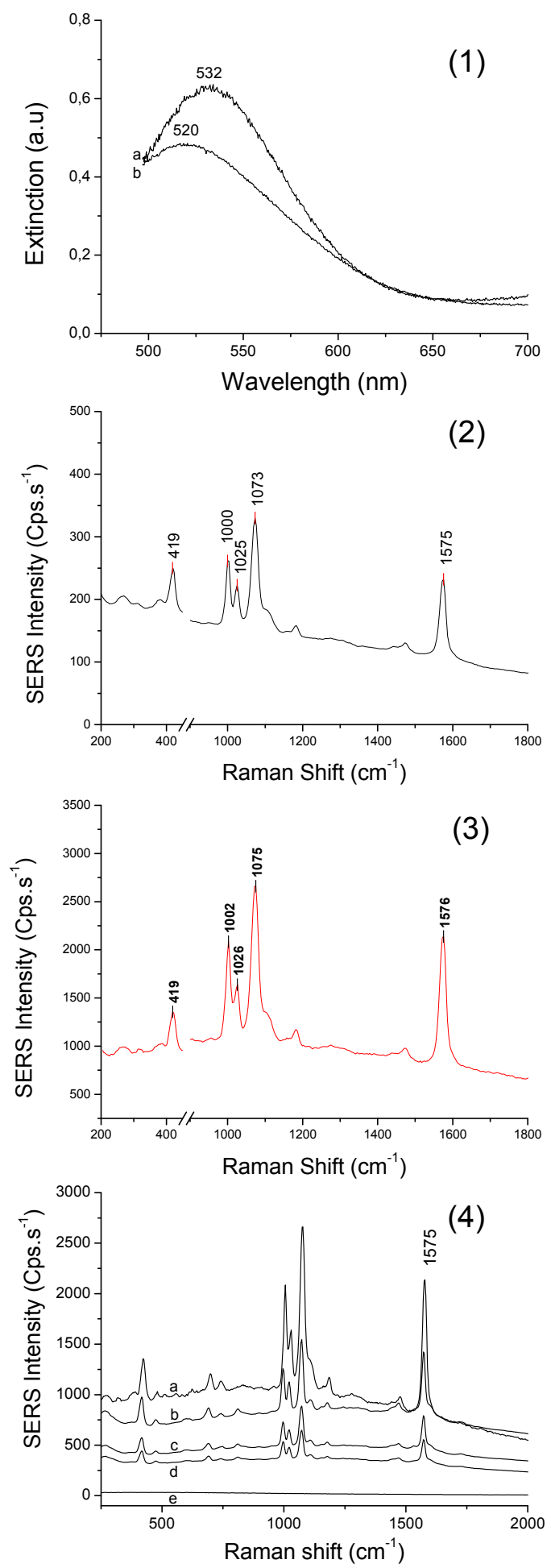

Fig. 3 (1) Extinction spectra of nanodisks for: (a) $D=50$ $\mathrm{nm}, \Lambda=80 \mathrm{~nm}, \lambda_{\text {Plasmon }}=520 \mathrm{~nm}$, and (b) $D=110$ $\mathrm{nm}, \Lambda=200 \mathrm{~nm}, \lambda_{\text {Plasmon }}=532 \mathrm{~nm}$. SERS results for nanodisks with the following sizes: (2) $D=50 \mathrm{~nm}$ and $\Lambda$ $=80 \mathrm{~nm}$, (3) $D=110 \mathrm{~nm}$ and $\Lambda=150 \mathrm{~nm}$. Characteristic Raman shifts are at $419 / 1000 / 1025 / 1075$ and $1575 \mathrm{~cm}^{-1}$. (4) SERS spectra for nanodisks with a diameter of $110 \mathrm{~nm}$ and four periods: (a) $150 \mathrm{~nm}$, (b) $200 \mathrm{~nm}$, (c) $250 \mathrm{~nm}$, and (d) $300 \mathrm{~nm}$, (e) no SERS signal is observed from the smooth gold film. where $I_{S E R S}, I_{\text {Raman }}$ are the SERS and Raman intensities, respectively. $N_{S E R S}, N_{\text {Raman }}$ are the number of excited molecules in SERS and Raman experiments, respectively. $N_{S E R S}$ depends on the numerical aperture and the laser spot size. It also depends on the periodicity and the size of the nanoparticles. $N_{S E R S}$ is calculated from the following equation:

$N_{\text {SERS }}=N_{A} \times S_{\text {Collected }} \times \sigma_{\text {Surf }} \times \frac{S_{\text {Structures }}}{\Lambda^{2}}$

where $N_{A}$ is the Avogadro's number $\left(\mathrm{mol}^{-1}\right)$, then $S_{\text {Collected }} \approx 144 \mu^{2}$ (see Section 2.3), $S_{\text {Structures }}$ is the sum of the lateral and top surfaces of the nanocylinder. $\sigma_{\text {Surf }}$ is the surface coverage of thiophenol, which is approximately $0.544 \mathrm{nmol} / \mathrm{cm}^{2}$ [21]. In the Raman experiment, the number of excited molecules $N_{\text {Raman }}$ is $4.22 \times 10^{15}$. This number is obtained by [22]: $N_{\text {Raman }}$ $=N_{A} \times C \times V$, where $C$ is the used concentration of Thiophenol $(1 M)$, and $V$ is the scattering volume and equal to $7 n L$. A maximum experimental $\mathrm{EF}$ value of $2.6 \times 10^{7}$ is found (see Table 1 ) for our system with the highest density (diameter of $110 \mathrm{~nm}$, and periodicity of $150 \mathrm{~nm}$ ). When the periodicity increases from $200 \mathrm{~nm}$ to $300 \mathrm{~nm}$, the EF value decreases from $2 \times$ $10^{7}$ to $1.5 \times 10^{7}$. Also, the standard deviation (DS) on a same substrate by taking into account the dose used for the lithography, is given in the table 1. The sampleto-sample variation has the same order of magnitude. We can directly link EF decreasing with the reduction of the nanodisk density.

\begin{tabular}{||c|c|c|c|c||}
\hline$D(\mathrm{~nm})$ & $\Lambda(\mathrm{nm})$ & $\rho(\%)$ & $\mathrm{EF}$ & $\mathrm{DS}(\%)$ \\
\hline 110 & 150 & 42.2 & $2.6 \times 10^{7}$ & 11.7 \\
110 & 200 & 23.8 & $2 \times 10^{7}$ & 9.1 \\
110 & 250 & 15.2 & $1.6 \times 10^{7}$ & 13.5 \\
110 & 300 & 10.6 & $1.5 \times 10^{7}$ & 15.4 \\
\hline 50 & 80 & 30.7 & $1.9 \times 10^{6}$ & 14.2 \\
\hline
\end{tabular}

Table 1 For the peak at $1575 \mathrm{~cm}^{-1}$, Enhancement Factor (EF) for different fill-factors $\rho$.

In addition, for small size of nanodisks (diameter of $50 \mathrm{~nm}$ and periodicity of $80 \mathrm{~nm}$ ), an EF of $1.9 \times$ $10^{6}$ is found. We suppose that this weaker value can be explained by the position of the experimental surface plasmon resonance $\left(\lambda_{\text {Plasmon }}=520 \mathrm{~nm}\right.$, see figure $3(1 \mathrm{a}))$ that is far from the excitation wavelength $\left(\lambda_{\text {exc }}\right.$ $=660 \mathrm{~nm}$ ) with such small nanoparticles, and also by the smaller density of this structure compared to the sample with $110 \mathrm{~nm}$ nanodisks (30.7 vs $42.2 \%$ ).

As shown elsewhere in the literature [23,24], the SERS intensity follows the structure local field enhancement at the excitation frequency and the Stokes shifted 
emission frequencies. This is the origin of the common $\left|E^{4}\right|$ approximation [25] for SERS enhancement factor. To estimate the SERS EF we therefore need to evaluate the near field intensity at the excitation and emission frequencies, respectively. Rigorous numerical calculations were carried out for this purpose to verify the trends observed in the experimental SERS EF. The numerical model $[15,26]$ that hybridizes two popular numerical methods namely the finite element method (FEM) and the Fourier modal method (FMM), was used for the calculation of near field intensities in the structures. Using these results, the SERS intensity distribution over the surface of the structure can be estimated as follows:

$I_{S E R S}=\beta \times I_{\lambda_{0}}(x, y, z) \times I_{\lambda_{R}}(x, y, z)$

where $\beta$ is a space independent constant and depends on the Raman tensor and thus on the chemical properties of the molecules used. $\mathrm{I}\left(\lambda_{0}\right)$ and $\mathrm{I}\left(\lambda_{R}\right)$ are the near field intensities $\left(E_{x}^{2}+E_{y}^{2}+E_{z}^{2}\right)$ at the excitation wavelength $\lambda_{0}$ and $\lambda_{R}$, respectively. In figure 4 , we show the SERS intensity distribution of the structures for periods of $150 \mathrm{~nm}$ and $300 \mathrm{~nm}$, considering plane wave incidence normal to the structures. The SERS intensity was normalized to the incident intensity $\left(I_{0}\right)$ for both the calculated intensities at the incident and emission wavelengths. We see that the SERS intensity decreases as the period of the structures increases as observed by the experimental study. To find the SERS Enhancement Factor of the structure, the previous local SERS intenisty must be integrated around the whole cylinder. The calculated SERS enhancement factor can be written as:

$E F_{E M} \approx \frac{1}{S} \times \iint_{S} I_{S E R S} d S$
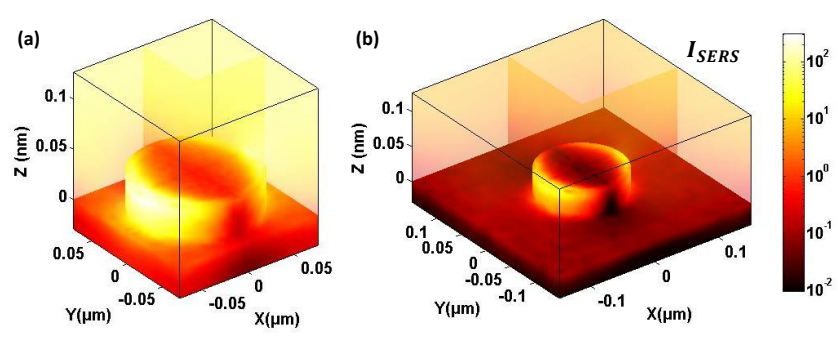

Fig. 4 The calculated SERS intensity distribution in log scale for two periods (a) $150 \mathrm{~nm}$ and (b) $300 \mathrm{~nm}$

The integral is normalized by the surface area over which the integration was carried out. The SERS inten- sity for two periods with the $110 \mathrm{~nm}$ diameter is displayed on figure 4 . To compare these results, we define simulated ratios $R_{\text {Field }}=E F_{E M_{110-X}} / E F_{E M_{110-300}}$ and experimental ratio $R_{E F}=E F_{110-X} / E F_{110-300}$ reported in Table 2 with the periodicity of $300 \mathrm{~nm}$ as reference and $X$ the studied period from $150 \mathrm{~nm}$ to $300 \mathrm{~nm}$.

\begin{tabular}{||c|c|c|c|}
\hline$D(\mathrm{~nm})$ & $\Lambda(\mathrm{nm})$ & $R_{\text {Field }}$ & $R_{E F}$ \\
\hline 110 & 150 & 1.7 & 1.7 \\
110 & 200 & 1.1 & 1.3 \\
110 & 250 & 1.03 & 1.06 \\
110 & 300 & 1 & 1 \\
\hline
\end{tabular}

Table 2 For the peak at $1575 \mathrm{~cm}^{-1}$, comparison of the trend of calculated SERS electromagnetic field intensity and experimental SERS EF.

A good agreement between simulation and experiment is obtained on density effect of nanodisks.

\section{Conclusion}

In this paper, we demonstrated the density effect of gold nanodisks on the SERS intensity with different densities varying from $10.6 \%$ to $42.2 \%$ on a gold film. As expected, the highest density of nanodisks (42.2\%) gave the greatest value of $\mathrm{EF}\left(\mathrm{EF}=2.6 \times 10^{7}\right)$ due to a better coupling between nanodisks and the consequent increasing of the electric field intensity around them. These experimental results are confirmed by numerical calculations of the electromagnetic field intensity. In addition, the size, and shape of nanostructures are homogenous and reproducible on large pattern areas, which are important points for industrial applications.

Acknowledgements The authors acknowledge ANR P2N (ANR-12-NANO-0016) and the support of the French Government for partial funding of the project in which this work takes place. This work was partly supported by the French RENATECH network. IOGS/CNRS is also part of the European Network of Excellence in BioPhotonics, Photonics for Life, P4L.

\section{References}

1. Li W, Ding F, Hu J, Chou S Y (2011) Three-dimensional cavity nanoantenna coupled plasmonic nanodots for ultrahigh and uniform surface-enhanced Raman scattering over large area. Opt Express 19(5):3925-3936

2. Oh Y -J, Jeong K -H (2012) Glass Nanopillar Arrays with Nanogap-Rich Silver Nanoislands for Highly Intense Surface Enhanced Raman Scattering. Adv Mater 24(17):2234-2237 
3. Guillot N, Lamy de la Chapelle M (2012) The electromagnetic effect in surface enhanced Raman scattering: Enhancement optimization using precisely controlled nanostructures. Journal of Quantitative Spectroscopy and Radiative Transfer 113(18):51-63

4. Barbillon G, Hamouda F, Held S, Gogol P, Bartenlian B (2010) Gold nanoparticles by soft UV nanoimprint lithography coupled to a lift-off process for plasmonic sensing of antibodies. Microelectron Eng 87:1001-1004

5. Hamouda F, Sahaf H, Held S, Barbillon G, Gogol P, Moyen E, Aassime A, Moreau J, Canva M, Lourtioz J -M, Hanbücken M, Bartenlian B (2011) Large area nanopatterning by combined anodic aluminum oxide and soft UVNIL technologies for applications in biology. Microelectron Eng 88:2444-2446

6. Lee S Y, Jeon H C, Yang S M (2012) Robust plasmonic sensors based on hybrid nanostructures with facile tunability. J Mater Chem 22(28):5900-5913

7. Camden J P, Dieringer J, Zhao J, Van Duyne R P (2008) Controlled Plasmonic Nanostructures for SurfaceEnhanced Spectroscopy and Sensing. Acc Chem Res 41(12):1653-1661

8. McFarland A D, Young M A, Dieringer J A, Van Duyne R P (2005) Wavelength-scanned surface-enhanced Raman excitation spectroscopy. J Phys Chem B 109(22):1127911285

9. Barbillon G, Bijeon J L, Plain J, Lamy de la Chapelle M, Adam P M, Royer P (2007) Electron beam lithography designed chemical nanosensors based on localized surface plasmon resonance. Surf Sci 601:5057-5061

10. Yu Q, Guan P, Qin D, Golden G, Wallace P M (2008) Inverted size-dependence of surface-enhanced Raman scattering on gold nanohole and nanodisk arrays. Nano Letters 8(7):1923-1928

11. Brown R J C, Milton M J T (2008) Nanostructures and nanostructured substrates for surface-enhanced Raman scattering (SERS). Journal of Raman Spectroscopy 39:1313-1326

12. Yu Q M, Braswell S, Christin B, Xu J J, Wallace P M, Gong H, Kaminsky D (2010) Surface-enhanced Raman scattering on gold quasi-3D nanostructure and 2D nanohole arrays. Nanotechnology 21(35):355301

13. Gutierrez-Rivera L, Peters R F, Dew S K, Stepanova M (2013) Application of EBL fabricated nanostructured substrates for surface enhanced Raman spectroscopy detection of protein A in aqueous solution. Journal of Vacuum Science and Technology B 31(6):06F901

14. Zhang P, Yang S, Wang L, Zhao J, Zhu Z, Liu B, Zhong J, Sun X (2014) Large-scale uniform Au nanodisk arrays fabricated via x-ray interference lithography for repro-ducible and sensitive SERS substrate. Nanotechnology 25(24):245301

15. Sarkar M, Besbes M, Moreau J, Bryche J F, Olivero A, Barbillon G, Coutrot A L, Bartenlian B, Canva M (2015) Hybrid Plasmonic Mode by Resonant Coupling of Localized Plasmons to Propagating Plasmons in a Kretschmann Configuration. ACS Photonics 2(2):237245

16. Carron K T, Hurley L G (1991) Axial and azimuthal angle determination with surface-enhanced Ramanspectroscopy - Thiophenol on copper, silver, and gold metal-surfaces. J Phys Chem 95(24):9979-9984

17. Gao H, McMahon J M, Lee M H, Henzie J, Gray S K, Schatz G C, Odom T W (2009) Rayleigh anomaly-surface plasmon polariton resonances in palladium and gold subwavelength hole arrays. Opt Express 17(4):2334-2340
18. McMahon J M, Li S, Ausman L K, Schatz G C (2012) Modeling the Effect of Small Gaps in Surface-Enhanced Raman Spectroscopy. J Phys Chem C 116(2):1627-1637

19. Sharma B, Frontiera R R, Henry A-I, Ringe E, Van Duyne R P (2012) SERS: Materials, applications, and the future. Materials Today 15(1-2):16-25

20. Barchiesi D, Kessentini S, Guillot N, de la Chapelle M L, Grosges T (2013) Localized surface plasmon resonance in arrays of nano-gold cylinders: inverse problem and propagation of uncertainties. Opt. Express 21(2):2245-2262

21. Caldwell J D, Glembocki O, Bezares F J, Bassim N D, Rendell R W, Feygelson M, Ukaegbu M, Kasica R, Shirey L, Hosten C (2011) Plasmonic Nanopillar Arrays for Large-Area, High-Enhancement Surface-Enhanced Raman Scattering Sensors. ACS Nano 5(5):4046-4055

22. Cottat M, Lidgi-Guigui N, Tijunelyte I, Barbillon G, Hamouda F, Gogol P, Aassime A, Lourtioz J M, Bartenlian B, Lamy de la Chapelle M (2014) Soft UV nanoimprint lithography-designed highly sensitive substrates for SERS detection. Nanoscale Research Letters 9:623

23. Le Ru E C, Etchegoin P G (2013) Quantifying SERS enhancements. MRS Bulletin 38(8):631-640

24. Saikin S K, Chu Y Z, Rappoport D, Crozier K B, Aspuru-Guzik A (2010) Separation of Electromagnetic and Chemical Contributions to Surface-Enhanced Raman Spectra on Nanoengineered Plasmonic Substrates. J Phys Chem Lett 1(18):2740-2746

25. Le Ru E C, Grand J, Felidj N, Aubard J, Levi G, Honenau A, Krenn J R, Blackie E, Etchegoin P G (2008) Experimental verification of the SERS electromagnetic model beyond the $|E|^{4}$ approximation: Polarization effects. J Phys Chem C 112(22):8117-8121

26. Hugonin J P, Besbes M, Lalanne P (2008) Hybridization of electromagnetic numerical methods through the G-matrix algorithm. Opt Letters 33(14):1590-1592 